\title{
Free energy and extension of a semiflexible polymer in cylindrical confining geometries
}

\author{
Yingzi Yang, ${ }^{1}$ Theodore W. Burkhardt, ${ }^{1,2}$ and Gerhard Gompper ${ }^{1}$ \\ ${ }^{1}$ Institut für Festkörperforschung, Forschungszentrum Jülich, D-52425 Jülich, Germany \\ ${ }^{2}$ Department of Physics, Temple University, Philadelphia, Pennsylvania 19122, USA
}

(Received 14 April 2007; published 19 July 2007)

\begin{abstract}
We consider a long, semiflexible polymer with persistence length $P$ and contour length $L$ fluctuating in a narrow cylindrical channel of diameter $D$. In the regime $D \ll P \ll L$ the free energy of confinement $\Delta F$ and the length of the channel $R_{\|}$occupied by the polymer are given by Odijk's relations $\Delta F / R_{\|}=A_{\circ} k_{B} T P^{-1 / 3} D^{-2 / 3}$ and $R_{\|}=L\left[1-\alpha_{\circ}(D / P)^{2 / 3}\right]$, where $A_{\circ}$ and $\alpha_{\circ}$ are dimensionless amplitudes. Using a simulation algorithm inspired by the pruned enriched Rosenbluth method, which yields results for very long polymers, we determine $A_{\circ}$ and $\alpha_{\circ}$ and the analogous amplitudes for a channel with a rectangular cross section. For a semiflexible polymer confined to the surface of a cylinder, the corresponding amplitudes are derived with an exact analytic approach. The results are relevant for interpreting experiments on biopolymers in microchannels or microfluidic devices.

DOI: $10.1103 /$ PhysRevE.76.011804

PACS number(s): 82.35.Lr, 87.15.Aa, 05.40.-a
\end{abstract}

\section{INTRODUCTION}

Microfluidic devices provide new possibilities for studying biological polymers such as DNA, actin filaments, and microtubules. Since the persistence lengths of biological polymers are typically tens of nanometers or larger, their behavior in confinement, as in nanochannels or microchannels, is different from that of flexible synthetic macromolecules.

In this paper we consider the equilibrium statistics of a semiflexible polymer or wormlike chain with persistence length $P$ and contour length $L$ in a cylindrical channel of diameter $D$. Here $D$ is an effective diameter, equal to twice the maximum transverse displacement of the polymer from the symmetry axis of the channel. For a channel with hard wall diameter $d_{c}$ containing a polymer with hard wall diameter $d_{p}, D=d_{c}-d_{p}$.

In the regime $D \ll P \ll L$, corresponding to a long, tightly confined polymer, Odijk [1] showed that the free energy of confinement $\Delta F$-i.e., the work required to reversibly insert the polymer in the channel-and the length of the channel, $R_{\|}$, occupied by the polymer are given by

$$
\begin{gathered}
\frac{\Delta F}{R_{\|}}=A_{\circ} \frac{k_{B} T}{P^{1 / 3} D^{2 / 3}}, \\
R_{\|}=L\left[1-\alpha_{\circ}\left(\frac{D}{P}\right)^{2 / 3}\right] .
\end{gathered}
$$

For a channel with a rectangular cross section with edges $D_{x}$ and $D_{y}$,

$$
\begin{aligned}
& \frac{\Delta F}{R_{\|}}=A_{\square} \frac{k_{B} T}{P^{1 / 3}}\left(\frac{1}{D_{x}^{2 / 3}}+\frac{1}{D_{y}^{2 / 3}}\right), \\
& R_{\|}=L\left(1-\alpha_{\square} \frac{D_{x}^{2 / 3}+D_{y}^{2 / 3}}{P^{2 / 3}}\right) .
\end{aligned}
$$

Here $A_{\circ}, \alpha_{\circ}, A_{\square}$, and $\alpha_{\square}$ are dimensionless universal numbers, which do not depend on $P, D, D_{x}$, and $D_{y}$.

Making use of advances in the manipulation of single polymers, recent experiments have begun to approach the
Odijk regime $D \ll P \ll L$. In the experiments of Reisner et al. [2] on single DNA molecules with persistence length $P$ of about $50 \mathrm{~nm}$ and contours lengths $L$ of around $20 \mu \mathrm{m}$ or larger, the condition $P \ll L$ is well satisfied, and the dimensions $D_{x}=30 \mathrm{~nm}$ and $D_{y}=40 \mathrm{~nm}$ of the narrowest channels are moderately smaller than $P$. In the experiments of Köster et al. [3] on actin filaments with persistence length of about $20 \mu \mathrm{m}$ in microchannels with diameters down to 1 or $2 \mu \mathrm{m}$, $D \ll P$ for the narrowest channels, and the longest contour lengths $L$ considered of around $50 \mu \mathrm{m}$ are about 2-3 times $P$. For an experiment in which DNA is confined by a grooved substrate instead of a channel, see Hochrein et al. [4].

For interpreting such experiments it is important to know the numerical values of the dimensionless amplitudes in Eqs. (1)-(4). Solving an integral equation numerically which arises in an exact analytic approach, Burkhardt [5] found

$$
A_{\square}=1.1036,
$$

and from simulations Bicout and Burkhardt [6] obtained

$$
A_{\square}=1.108 \pm 0.013, \quad A_{\circ}=2.375 \pm 0.013 .
$$

Other estimates from simulations, compatible with these values but with larger error bars, are given in Refs. [7-9] and related results for a helical polymer in a cylindrical channel in Ref. [10].

Although the free-energy amplitudes $A_{\square}$ and $A_{\circ}$ are known with good precision, comparable estimates of the extension amplitudes $\alpha_{\square}$ and $\alpha_{\circ}$ have not been available. Thus, we have determined $\alpha_{\square}$ and $\alpha_{\circ}$ from simulations and obtained more precise estimates of $A_{\square}$ and $A_{\circ}$, as described in this paper.

The surface of a channel can be prepared so that biopolymers are adsorbed. For example, naturally anionic DNA strands are adsorbed on a surface coated with cationic lipid membranes and have a high lateral mobility on the surface $[4,13,14]$. The attractive interaction between the surface and the biopolymer lowers the free-energy barrier for insertion of a macromolecule in a narrow channel. With this as motivation we also consider the free energy and extension of a semiflexible polymer confined to the surface of a cylinder. 


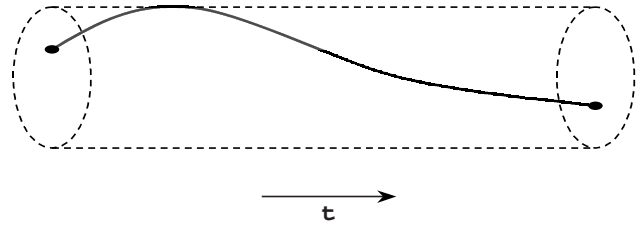

FIG. 1. The curve may be interpreted as a tightly confined semiflexible polymer in a channel with a circular cross section or as the trajectory $\vec{r}(t)$, plotted as a function of $t$, of a randomly accelerated particle moving in two dimensions, which has not yet left a circular domain.

The theoretical framework for our calculations is outlined in Sec. II. In Sec. III a simulation algorithm inspired by the pruned enriched Rosenbluth method (PERM) [15-17] is described, which enables us to consider polymers two or more orders of magnitude longer than in the simulations of Ref. [6], on which the results (6) are based. Our estimates of the amplitudes $A_{\square}, A_{\circ}, \alpha_{\square}$, and $\alpha_{\circ}$ are given in Sec. III. In Sec. IV we consider a semiflexible polymer confined to the surface of a cylinder with diameter $D$. In the regime $D \ll P$ $\ll L$ the confinement free energy and extension are also given by Eqs. (1) and (2), but with different amplitudes $A_{\mathcal{S}}$ and $\alpha_{\mathcal{S}}$. These amplitudes are calculated with an exact analytic approach. In the concluding section $\mathrm{V}$ we compare the results of Secs. III and IV with predictions for a polymer confined by an effective parabolic potential.

\section{THEORETICAL FRAMEWORK}

In the wormlike chain model of a semiflexible polymer, the bending energy is given by

$$
\mathcal{H}=\frac{\kappa}{2} \int_{0}^{L} d s\left(\frac{d \hat{\tau}}{d s}\right)^{2} .
$$

Here $\hat{\tau}$ is the unit vector tangent to the polymer contour, $s$ is the arclength, and $\kappa$ is the bending rigidity, related to the persistence length by $P=\kappa / k_{B} T$. In the regime $D \ll P \ll L$, backfolding of the polymer-i.e., configurations with "hairpins" [18] — and excluded-volume effects are negligible. In typical polymer configurations the tangent vector is nearly parallel to the symmetry axis of the channel. The configurations correspond to single-valued functions $\vec{r}(t)$, where $(x, y, t)$ are Cartesian coordinates (see Fig. 1) and $\vec{r}=(x, y)$ specifies the transverse displacement of the polymer from the symmetry axis or $t$ axis of the channel. Since $|\vec{v}| \ll 1$, where $\vec{v}=d \vec{r} / d t$, the bending energy (7) simplifies to

$$
\mathcal{H}=\frac{\kappa}{2} \int_{0}^{L} d t\left(\frac{d^{2} \vec{r}}{d t^{2}}\right)^{2}
$$

and the length of the channel $R_{\|}$occupied by the polymer and the contour length $L$ are related by

$$
L=\int_{0}^{R_{\|}} d t\left(1+\vec{v}^{2}\right)^{1 / 2} \approx R_{\|}+\frac{1}{2} \int_{0}^{R_{\|}} d t \vec{v}^{2} .
$$

The partition function of a polymer in a channel, with position and slope $\vec{r}_{0}, \vec{v}_{0}$ at $t=0$ and $\vec{r}, \vec{v}$ at $t$, with bending energy (8), and without backfolding, is given by the path integral

$$
Z\left(\vec{r}, \vec{v} ; \vec{r}_{0}, \vec{v}_{0} ; t\right)=\int D^{2} r \exp \left[-\frac{P}{2} \int_{0}^{t} d t\left(\frac{d^{2} \vec{r}}{d t^{2}}\right)^{2}\right],
$$

where the paths are restricted to the interior of the channel. It satisfies the Fokker-Planck type differential equation

$$
\left(\frac{\partial}{\partial t}+\vec{v} \cdot \vec{\nabla}_{\mathbf{r}}-\frac{1}{2 P} \nabla_{\mathbf{v}}^{2}\right) Z\left(\vec{r}, \vec{v} ; \vec{r}_{0}, \vec{v}_{0} ; t\right)=0,
$$

with the initial condition $Z\left(\vec{r}, \vec{v} ; \vec{r}_{0}, \vec{v}_{0} ; 0\right)=\delta\left(\vec{r}-\vec{r}_{0}\right) \delta\left(\vec{v}-\vec{v}_{0}\right)$.

The boundary condition at a "hard" channel wall follows from the fact that discontinuities in the slope of the polymer cost an infinite bending energy and are suppressed. Thus, as $\vec{r}$ approaches the channel wall, $Z\left(\vec{r}, \vec{v} ; \vec{r}_{0}, \vec{v}_{0} ; t\right)$ vanishes for $\hat{n} \cdot \vec{v}>0$, but not for $\hat{n} \cdot \vec{v}<0$, where $\hat{n}$ is normal to the wall and directed toward the interior of the channel [5].

Our reason for denoting the Cartesian coordinates by $(x, y, t)$ instead of $(x, y, z)$ is explained in Fig. 1. Each polymer configuration $\vec{r}(t)$ may be interpreted as the position of a randomly accelerated particle in two dimensions, plotted as a function of the time $t$. The polymer partition function (10) corresponds to the propagator or probability density for propagation from initial position and velocity $\vec{r}_{0}, \vec{v}_{0}$ to $\vec{r}, \vec{v}$ in a time $t$. From the Boltzmann factor in Eq. (10) one sees that the acceleration of the particle at each instant is an independent, Gaussian-distributed random variable, with

$$
\frac{d^{2} \vec{r}}{d t^{2}}=\vec{\eta}(t), \quad\langle\vec{\eta}(t)\rangle=0, \quad\left\langle\eta_{i}(t) \eta_{j}\left(t^{\prime}\right)\right\rangle=\frac{\delta_{i j}}{P} \delta\left(t-t^{\prime}\right) .
$$

Since the polymer partition function vanishes at a hard wall for $\hat{n} \cdot \vec{v}>0$, the propagator for the randomly accelerated particle vanishes if the particle is reflected toward the interior of the two-dimensional domain representing the channel cross section. Thus, the hard wall in the polymer problem corresponds to an absorbing boundary for the randomly accelerated particle.

For large $t$ the partion function (10) decays as

$$
Z\left(\vec{r}, \vec{v} ; \vec{r}_{0}, \vec{v}_{0} ; t\right) \approx \psi_{0}(\vec{r}, \vec{v}) \psi_{0}\left(\vec{r}_{0},-\vec{v}_{0}\right) e^{-E_{0}(P, D) t}, \quad t \rightarrow \infty,
$$

where $E_{0}(P, D)$ is the smallest eigenvalue of the $t$ independent Fokker-Planck equation

$$
\left[-E(P, D)+\vec{v} \cdot \vec{\nabla}_{\mathbf{r}}-\frac{1}{2 P} \nabla_{\mathbf{v}}^{2}\right] \psi(\vec{r}, \vec{v})=0 .
$$

Together with the definition

$$
\exp \left(-\frac{\Delta F}{k_{B} T}\right)=\frac{Z(D)}{Z(\infty)}
$$

of the free energy of confinement, Eq. (13) implies 


$$
\frac{\Delta F}{k_{B} T R_{\|}}=E_{0}^{\circ}(P, D)-E_{0}^{\circ}(P, \infty)=\frac{E_{0}^{\circ}\left(\frac{1}{2}, 1\right)}{(2 P)^{1 / 3} D^{2 / 3}}
$$

for a channel with a circular cross section, in agreement with Odijk's relation (1). Here we have used the scaling relation $E_{0}^{\circ}(P, D)=(2 P)^{-1 / 3} D^{-2 / 3} E_{0}^{\circ}\left(\frac{1}{2}, 1\right)$, which is readily derived by rewriting Eq. (14) in terms of the dimensionless variables $\vec{r}^{\prime}=D^{-1} \vec{r}, t^{\prime}=(2 P)^{-1 / 3} D^{-2 / 3} t$, and $\vec{v}^{\prime}=(2 P)^{1 / 3} D^{-1 / 3} \vec{v}$.

For a channel with a rectangular cross section with edges $D_{x}$ and $D_{y}$, the partition function in Eq. (10) has the product form $Z\left(\vec{r}, \vec{v} ; \vec{r}_{0}, \vec{v}_{0} ; t\right)=Z\left(x, v_{x} ; x_{0}, v_{x 0} ; t\right) Z\left(y, v_{y} ; y_{0}, v_{y 0} ; t\right)$. This is the origin of the sum of independent $x$ and $y$ contributions in Eqs. (3) and (4). The solutions to Eq. (14) also have the separable form $\psi(\vec{r}, \vec{v})=\psi\left(x, v_{x},\right) \psi\left(y, v_{y}\right)$, implying $E_{0}^{\square}\left(P, D_{x}, D_{y}\right)=E_{0}^{\rfloor}\left(P, D_{x}\right)+E_{0}^{\rfloor}\left(P, D_{y}\right)$. Here $E_{0}^{\rfloor}\left(P, D_{x}\right)$ is the smallest eigenvalue of the equation

$$
\left[-E^{\mid}(P, D)+v_{x} \frac{\partial}{\partial x}-\frac{1}{2 P} \frac{\partial^{2}}{\partial v_{x}^{2}}\right] \psi\left(x, v_{x}\right)=0
$$

on the one-dimensional interval $-\frac{1}{2} D_{x}<x<\frac{1}{2} D_{x}$, with boundary condition $\psi\left(-\frac{1}{2} D_{x}, v_{x}\right)=\psi\left(\frac{1}{2} D_{x},-v_{x}\right)=0$ for $v_{x}>0$. The scaling relation $E_{0}^{\mid}\left(P, D_{x}\right)=(2 P)^{-1 / 3} D_{x}^{-2 / 3} E_{0}^{\mid}\left(\frac{1}{2}, 1\right)$, is readily derived by rewriting Eq. (17) in terms of the dimensionless variables $x^{\prime}=D^{-1} x, \quad t^{\prime}=(2 P)^{-1 / 3} D_{x}^{-2 / 3} t$, and $v_{x}^{\prime}$ $=(2 P)^{1 / 3} D_{x}^{-1 / 3} v_{x}$.

From Eqs. (1), (3), and (16) and the results of the preceding paragraph, we obtain

$$
A_{\circ}=2^{-1 / 3} E_{0}^{\circ}\left(\frac{1}{2}, 1\right), \quad A_{\square}=2^{-1 / 3} E_{0}\left(\frac{1}{2}, 1\right) .
$$

To obtain comparable expressions for the amplitudes $\alpha$ 。 and $\alpha_{\square}$, we begin by comparing Eqs. (2) and (9), which imply

$$
\alpha_{\circ}=\frac{1}{2}\left(\frac{P}{D}\right)^{2 / 3}\left\langle\vec{v}^{2}\right\rangle_{P, D}^{\circ}
$$

Here $\left\langle\vec{v}^{2}\right\rangle_{P, D}^{\circ}$ is the average value of $\vec{v}^{2}$ along an infinitely long, tightly confined polymer in a channel with a circular cross section. In terms of the ground-state eigenfunction $\psi_{0}(\vec{r}, \vec{v})$ of Eq. (14) with eigenvalue $E_{0}^{\circ}(P, D)$,

$$
\left\langle\vec{v}^{2}\right\rangle_{P, D}^{\circ}=\frac{\int d^{2} r \int d^{2} v \vec{v}^{2} \psi_{0}(\vec{r}, \vec{v}) \psi_{0}(\vec{r},-\vec{v})}{\int d^{2} r \int d^{2} v \psi_{0}(\vec{r}, \vec{v}) \psi_{0}(\vec{r},-\vec{v})} .
$$

Expressing Eq. (19) and its analog for the rectangular cross section in terms of the dimensionless variables introduced below Eqs. (16) and (17), we obtain

$$
\alpha_{\circ}=2^{-5 / 3}\left\langle\vec{v}^{\prime 2}\right\rangle_{1 / 2,1}^{\circ}, \quad \alpha_{\square}=2^{-5 / 3}\left\langle v_{x}^{\prime 2}\right\rangle_{1 / 2,1},
$$

Equations (18) and (21) play a central role in our work, allowing us to determine the free energy and extension amplitudes from simulations with the dimensionless parameters $D^{\prime}=D_{x}^{\prime}=D_{y}^{\prime}=2 P^{\prime}=1$. We emphasize that the approach for calculating the amplitudes $A_{\circ}, A_{\square}, \alpha_{\circ}$, and $\alpha_{\square}$ based on Eqs. (18) and (21) is exact, apart from statistical errors in the simulations. We always work in the regime where the unprimed parameters satisfy $D, D_{x}, D_{y} \ll P \ll L$ and the partition function of the wormlike chain reduces to the form (10).

\section{SIMULATIONS}

\section{A. Algorithm}

To determine $A_{\square}$ and $\alpha_{\square}$ from simulations, we generate a large number $N_{0}$ of configurations $x^{\prime}\left(t^{\prime}\right)$ of a polymer with persistence length $P^{\prime}=\frac{1}{2}$ in the unbounded two-dimensional space $\left(x^{\prime}, t^{\prime}\right)$. Here $x^{\prime}$ and $t^{\prime}$ are the dimensionless coordinates introduced below Eq. (17). The configurations are generated with the same Boltzmann weight as in Eq. (10), but in two rather than three spatial dimensions. The amplitudes $A_{\circ}$, $\alpha_{\circ}, A_{\square}$, and $\alpha_{\square}$ in Eqs. (1)-(4) are defined in the limit of infinite contour length and do not depend on the boundary conditions at the ends of the polymer. Thus, we use the same simple initial condition $x_{0}^{\prime}=v_{0}^{\prime}=0$ at $t_{0}^{\prime}=0$ for each configuration. Each configuration is "grown" until it leaves the interval $-\frac{1}{2}<x^{\prime}<\frac{1}{2}$ for the first time. From this information we calculate the fraction $Q\left(t^{\prime}\right)$ of the $N_{0}$ configurations which have not yet left the interval at $t^{\prime}$.

From Eq. (15) we see that $Q\left(t^{\prime}\right)=\exp \left(-\Delta F / k_{B} T\right)$, where $\Delta F$ is the free energy of confinement of a polymer with one end fixed, as described above, which extends a distance $t^{\prime}$ down a two-dimensional channel of width 1 . According to Eq. (13) and the discussion below Eq. (17), $Q\left(t^{\prime}\right)$ decays as

$$
Q\left(t^{\prime}\right) \sim e^{-E_{0}^{\mid}(1 / 2,1) t^{\prime}}
$$

for large $t^{\prime}$. To estimate $A_{\square}$, we fit the $Q\left(t^{\prime}\right)$ extracted from the simulations with the exponential form (22) for large $t^{\prime}$ to obtain $E_{0}^{(}\left(\frac{1}{2}, 1\right)$ and then use Eq. (18).

The polymer configurations are generated with the algorithm

$$
\begin{gathered}
x_{n+1}^{\prime}=x_{n}^{\prime}+v_{n}^{\prime} \Delta_{n+1}+\left(\frac{\Delta_{n+1}^{3}}{6}\right)^{1 / 2}\left(s_{n+1}+\sqrt{3} r_{n+1}\right), \\
v_{n+1}^{\prime}=v_{n}^{\prime}+\left(2 \Delta_{n+1}\right)^{1 / 2} r_{n+1},
\end{gathered}
$$

introduced in Ref. [11] and also used in Refs. [6,12]. Here $x_{n}^{\prime}$ is the position of the polymer at point $t_{n}^{\prime}$ and $\Delta_{n+1}=t_{n+1}^{\prime}-t_{n}^{\prime}$ is the length step. The quantities $r_{n}$ and $s_{n}$ are independent, Gaussian random numbers with $\left\langle r_{n}\right\rangle=\left\langle s_{n}\right\rangle=0$ and $\left\langle r_{n}^{2}\right\rangle=\left\langle s_{n}^{2}\right\rangle$ $=1$.

As discussed in Refs. [6,11,12], this algorithm generates polymer configurations consistent with the Boltzmann weight (10) in free space-i.e., in the absence of boundaries. An advantage of the algorithm is that in free space there is no length-step error. The length step $\Delta_{n+1}$ need not be small. For good efficiency we use a fairly large step when $x_{n}^{\prime}$ is well inside the interval $-\frac{1}{2}<x^{\prime}<\frac{1}{2}$. Near $x^{\prime}= \pm \frac{1}{2}$ a smaller step is needed in order to accurately determine the value of $t^{\prime}$ at which the configuration leaves the interval for the first time and hence $Q\left(t^{\prime}\right)$. As in Ref. [6], we choose

$$
\Delta_{n+1}=10^{-1}\left(\frac{1}{2}-|x|\right)+10^{-5},
$$

which varies from 0.05 at $x^{\prime}=0$ to $10^{-5}$ at $x^{\prime}= \pm \frac{1}{2}$. Further reduction of the length step had no significant effect on our estimates. 
In estimating $\left\langle v_{x}^{\prime 2}\right\rangle_{1 / 2,1}$ in Eq. (21) to determine $\alpha_{\square}$, one should only use the subset of the configurations, generated as described above, which lie entirely within the interval $-\frac{1}{2}$ $<x^{\prime}<\frac{1}{2}$-i.e., within the channel. For each of the configurations $\mathcal{C}$ in the subset we average $v^{\prime 2}$, over the entire length of each of the configurations in the subset, using

$$
\left\langle v^{\prime 2}\right\rangle_{\mathcal{C}}=\sum_{n} \frac{\Delta_{n+1}}{t^{\prime}}\left\langle v^{\prime 2}\right\rangle_{n+1}
$$

Here $t^{\prime}=\Sigma_{n} \Delta_{n+1}$ is the total length of the channel occupied by configuration $\mathcal{C}$ and $\left\langle v^{\prime 2}\right\rangle_{n+1}$ is the equilibrium value of $v^{\prime 2}$ for a semiflexible polymer with end points $\left(x_{n}^{\prime}, v_{n}^{\prime}\right)$ and $\left(x_{n+1}^{\prime}, v_{n+1}^{\prime}\right)$ at $t_{n}^{\prime}=$ and $t_{n+1}^{\prime}$, respectively, averaged over all intermediate $t^{\prime}$. This quantity is readily calculated from the free space partition function or propagator and is given by

$$
\begin{aligned}
\left\langle v^{\prime 2}\right\rangle_{n+1}= & \frac{2}{15} \Delta_{n+1}+\frac{6}{5}\left(x_{n+1}^{\prime}-x_{n}^{\prime}\right)^{2} \Delta_{n+1}^{-2}+\frac{2}{15}\left(v_{n+1}^{\prime 2}-\frac{1}{2} v_{n+1}^{\prime} v_{n}^{\prime}\right. \\
& \left.+v_{n}^{\prime 2}\right)-\frac{1}{5}\left(x_{n+1}^{\prime}-x_{n}^{\prime}\right)\left(v_{n+1}^{\prime}+v_{n}^{\prime}\right) \Delta_{n+1}^{-1}
\end{aligned}
$$

Having calculated $\left\langle v^{\prime 2}\right\rangle_{\mathcal{C}}$ for each configuration in the subset this way, we average the results over all the configurations in the subset to obtain an estimate of $\left\langle v_{x}^{\prime 2}\right\rangle_{1 / 2,1}$ and, using Eq. (21), the corresponding value of $\alpha_{\square}$.

\section{B. Enrichment procedure}

The quantity $\left\langle v_{x}^{\prime 2}\right\rangle_{1 / 2,1}$ in Eq. (21) is the average value of $v^{2}$ for a semiflexible polymer of infinite length in a channel. We found it necessary to go to lengths $t^{\prime}$ of around 100 to estimate $\left\langle v_{x}^{\prime 2}\right\rangle_{1 / 2,1}$, free of finite-length effects, to three significant figures. However, it is not feasible to generate configurations this long, which lie entirely within the channel, without modifying the steps outlined in the preceding paragraphs. From Eqs. (5) and (21), $E_{0}\left(\frac{1}{2}, 1\right)$ is close to 1.390. Thus, according to Eq. (22), the probability that a configuration of length $t^{\prime}=100$, generated as described above, never leaves the channel $-\frac{1}{2}<x^{\prime}<\frac{1}{2}$ is about $e^{-139} \approx 10^{-61}$.

To generate a large, statistically useful number of configurations lying entirely in the channel, we used an enrichment procedure inspired by PERM [15-17], which has been successfully applied in simulations of a wide variety of systems, including flexible, self-avoiding polymers in channels $[19,20]$.

We begin by generating a large number $N_{0}$ of configurations as described above. Let $N_{1}$ be the number of these configurations which have not yet left the channel at $t^{\prime}=\tau$. We make $n$ copies of each of these configurations and then, with the algorithm of the preceding subsection, continue each of the $n N_{1}$ configurations past $t^{\prime}=\tau$. Let $N_{2}$ be the number of these configurations which have not yet left the channel at $t^{\prime}=2 \tau$. Again we make $n$ copies and then continue the $n N_{2}$ configurations past $t^{\prime}=2 \tau$. At $t^{\prime}=3 \tau, 4 \tau, \ldots$, the same procedure is followed.

To estimate $A_{\square}$ using Eqs. (18) and (22), we need to calculate the probability $Q\left(t^{\prime}\right)$, defined above Eq. (22), that a configuration, generated as in the preceding subsection, has not yet left the channel at $t^{\prime}$. To obtain this probability, it is useful to think of copying all preceding configurations, including the number of initial configurations, at $t^{\prime}=\tau, 2 \tau, \ldots$. Thus,

$$
Q(0)=1, \quad Q(k \tau)=\frac{N_{k}}{n^{k-1} N_{0}} \text { for } k=1,2, \ldots
$$

Our results for a semiflexible polymer in two dimensions were obtained with $N_{0}=1.8 \times 10^{7}, \tau=1$, and $n=4$. These values of $n$ and $\tau$ were chosen so that $N_{k}$ slowly decreases with increasing $k$. From Eqs. (5), (18), (22), and (28), one finds $N_{k} \sim n^{k} e^{-E_{0}(1 / 2,1) k \tau} N_{0}=(0.996)^{k} N_{0}$.

We have also calculated the number of families, $N_{k}^{\mathrm{fam}}$, to which the $N_{k}$ configurations that remain in the channel up to $t^{\prime}=k \tau$ belong. Two configurations are said to belong to the same family if they coincide in the interval $0<t^{\prime}<\tau$-i.e., if their most remote ancestor is the same. By definition $N_{1}^{\mathrm{fam}}$ $=N_{1}$, but for larger $k, N_{k}^{\mathrm{fam}} \leqslant N_{k}$, since several of the $N_{k}$ configurations may belong to the same family. According to our simulation data $N_{k}^{\text {fam }}$ also decays as $n^{k} e^{-E_{0}(1 / 2,1) k \tau}=(0.996)^{k}$. For sufficiently large $k$ all $N_{k}$ configurations belong to a single family.

To determine $\alpha_{\square}$, we evaluate $\left\langle v_{x}^{\prime 2}\right\rangle_{1 / 2,1}$, as outlined above in the paragraph containing Eqs. (26) and (27), for those $N_{k}$ configurations which remain in the channel up to $t^{\prime}=k \tau$, estimate the limiting value for large $t^{\prime}$, and then use Eq. (21).

The simulations of a polymer in a channel with a circular cross section of diameter $D$ are very similar. In terms of the dimensionless Cartesian coordinates $\left(x^{\prime}, y^{\prime}, t^{\prime}\right)$ introduced below Eq. (16), the channel has radius $\frac{1}{2}$. Beginning with $x_{0}^{\prime}=y_{0}^{\prime}=v_{x 0}^{\prime}=v_{y 0}^{\prime}=t_{0}^{\prime}=0$, we generate the sequence $\left(x_{n}^{\prime}, y_{n}^{\prime}, t_{n}^{\prime}\right)$ with the algorithm (23) and (24) and corresponding equations with $x$ replaced by $y$. In analogy with Eq. (25) the length step is

$$
\Delta_{n+1}=10^{-1}\left[\frac{1}{2}-\left(x_{n}^{\prime 2}+y_{n}^{\prime 2}\right)^{1 / 2}\right]+10^{-5} .
$$

Each configuration is grown until it leaves the circular domain $\left(x^{\prime 2}+y^{\prime 2}\right)^{1 / 2}<\frac{1}{2}$. Again we begin with $N_{0}$ configurations and at $t^{\prime}=\tau, 2 \tau, \ldots$ make $n$ copies of the $N_{1}, N_{2}, \ldots$ configurations which have not yet left the circular domain. Our results were obtained with $N_{0}=4.7 \times 10^{7}, \tau=1.009$, and $n=20$. As in the two-dimensional case these parameters were chosen so that $N_{k}$ and $N_{k}^{\text {fam }}$ decay rather slowly, as $n^{k} e^{-E_{0}^{\circ}(1 / 2,1) k \tau}=(0.997)^{k}$, where we have used Eqs. (18), (22), and (28) and our result for $A_{\circ}$ in Eq. (30).

To estimate $A_{\circ}$, we calculate $Q\left(t^{\prime}\right)$ for integer $t^{\prime}$ using Eq. (28), fit the results with the exponential form (22), but with $E_{0}^{\circ}\left(\frac{1}{2}, 1\right)$ in place of $E_{0}^{\mid}\left(\frac{1}{2}, 1\right)$, and then use Eq. (18). To estimate $\alpha_{\circ}$, we evaluate $\left\langle\vec{v}^{\prime 2}\right\rangle_{1 / 2,1}^{\circ}$, as described in connection with Eqs. (26) and (27), for those $N_{k}$ configurations which remain in the channel up to $t^{\prime}=k \tau$ and then use Eq. (21).

\section{Results}

In Fig. 2, $\ln Q\left(t^{\prime}\right)$, as determined from Eq. (28), is shown for $t^{\prime}=0, \tau, 2 \tau, \ldots$ for a polymer on a two-dimensional strip 


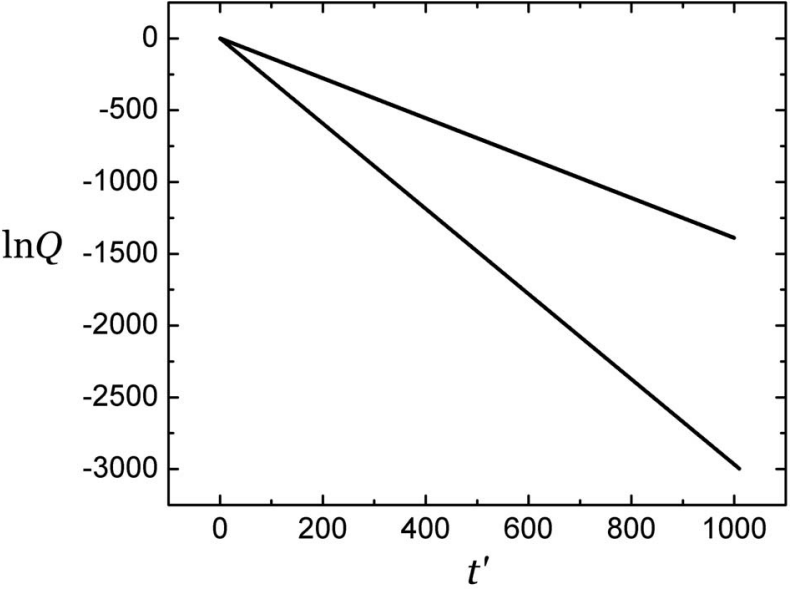

FIG. 2. $\ln Q\left(t^{\prime}\right)$ vs $t^{\prime}$ for a polymer on a two-dimensional strip (upper curve) and for a polymer in a three-dimensional channel with a circular cross section (lower curve).

(upper curve) and in a three-dimensional channel with a circular cross section (lower curve). According to Eqs. (22) and (18) the upper and lower curves have slope $E_{0}^{\dagger}\left(\frac{1}{2}, 1\right)$ $=2^{1 / 3} A_{\square}$ and $E_{0}^{\circ}\left(\frac{1}{2}, 1\right)=2^{1 / 3} A_{\circ}$, respectively, for large $t^{\prime}$. From the best fit to the slope, we obtain

$$
A_{\square}=1.1038 \pm 0.0006, \quad A_{\circ}=2.3565 \pm 0.0004 .
$$

The uncertainty was estimated by determining $A_{\square}$ and $A_{\circ}$ in each of the intervals $100<t^{\prime}<200,200<t^{\prime}<300, \ldots, 900$ $<t^{\prime}<1000$ and quoting a value somewhat larger than the width of the corresponding distribution.

The estimate for $A_{\square}$ is in extremely good agreement with the result in Eq. (5), obtained by solving an integral equation numerically that determines $A_{\square}$ in an exact analytic approach [5]. The new estimates for $A_{\square}$ and $A_{\circ}$ in Eq. (30) have smaller error bars than the earlier estimates [6] shown in Eq. (6), which are based on simulations of much shorter polymer chains.

In Fig. 3 the $t^{\prime}$ dependence of $\left\langle v_{x}^{\prime 2}\right\rangle_{1 / 2,1}$ and $\left\langle\vec{v}^{\prime 2}\right\rangle_{1 / 2,1}^{\circ}$ is shown. The averages are based on the configurations which remain in the channel from the starting point up to $t^{\prime}$. For $t^{\prime}$ greater than roughly 100 the curves are consistent, within statistical fluctuations, with the constant values $\left\langle v_{x}^{\prime 2}\right\rangle_{1 / 2,1}^{\prime}$ $=0.2901 \pm 0.0003$ and $\left\langle\vec{v}^{\prime 2}\right\rangle_{1 / 2,1}^{\circ}=0.5400 \pm 0.0004$. Substituting these values in Eq. (21), we obtain

$$
\alpha_{\square}=0.09137 \pm 0.00007, \quad \alpha_{\circ}=0.1701 \pm 0.0001 .
$$

For a polymer on a two-dimensional strip, the probability distribution $P\left(\left\langle v^{\prime 2}\right\rangle\right)$ of the quantity $\left\langle v^{\prime 2}\right\rangle$ is shown for representative values of $t^{\prime}$ in Fig. 4. The distribution was determined from the results for $\left\langle v^{\prime 2}\right\rangle_{\mathcal{C}}$, where the index $\mathcal{C}$ labels the configurations that remain in the channel up to length $t^{\prime}$ and $\left\langle v^{\prime 2}\right\rangle_{\mathcal{C}}$ is the average value of $v^{\prime 2}$ along configuration $\mathcal{C}$ from the starting point up to $t^{\prime}$, calculated as in Eqs. (26) and (27). The distributions in Fig. 4, are approximately Gaussian, and the half width or standard deviation $w$, shown in Fig. 5, decreases in good agreement with the $t^{\prime-1 / 2}$ law expected for statistically independent contributions. Results similar to

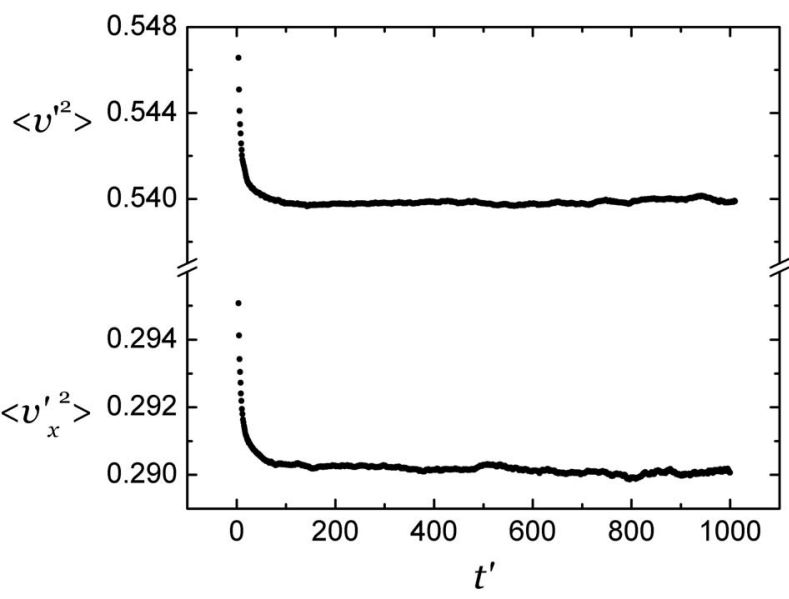

FIG. 3. $\left\langle v_{x}^{\prime 2}\right\rangle_{1 / 2,1}$ vs $t^{\prime}$ for a polymer on a two-dimensional strip (lower curve) and $\left\langle\vec{v}^{\prime 2}\right\rangle_{1 / 2,1}^{\circ}$ vs $t^{\prime}$ for a polymer in a threedimensional channel with a circular cross section (upper curve).

those in Figs. 4 and 5 were also obtained for a polymer in a channel with a circular cross section.

Since $\left\langle v^{\prime 2}\right\rangle_{\mathcal{C}}$ determines the contour length $L^{\prime}$ of configuration $\mathcal{C}$ via Eq. (9), the curves in Fig. 4 may be interpreted as distributions of the contour length $L^{\prime}$ for fixed $t^{\prime}$. Presumably the distribution of $t^{\prime}$ for fixed $L^{\prime}$-i.e., the end-to-end distribution for a polymer of fixed contour length [21] — is very similar.

\section{SEMIFLEXIBLE POLYMER CONFINED TO A CYLINDRICAL SURFACE}

In this section we consider a semiflexible polymer constrained to lie on a cylindrical surface with a circular cross section. As mentioned in the Introduction, this is an obvious model for a semiflexible polymer adsorbed on a channel wall. We analyze the case in which only configurations that leave the polymer in contact with the cylindrical surface are

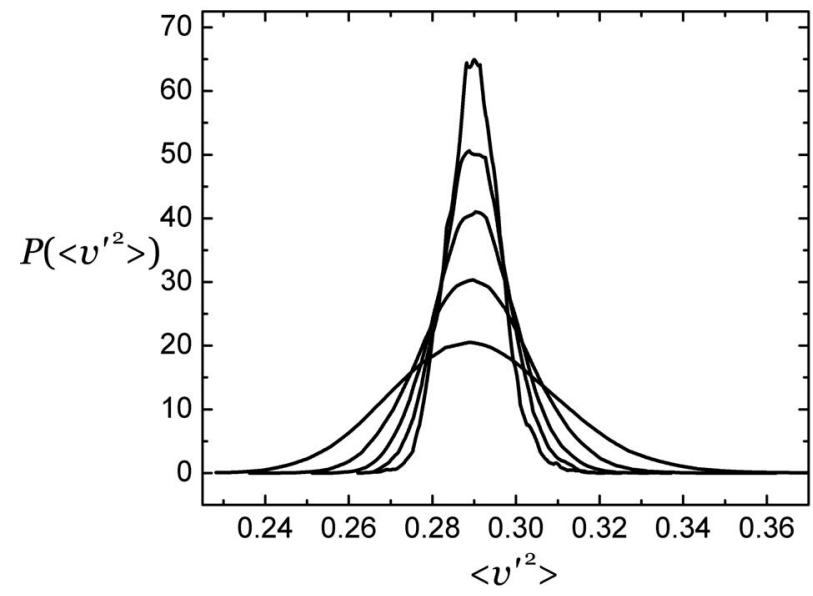

FIG. 4. Distribution $P\left(\left\langle v^{\prime 2}\right\rangle\right)$ for a polymer on a twodimensional strip, as defined below Eq. (31). The curves correspond, from bottom to top, to $t^{\prime}=100,225,400,625$, and 900 . 


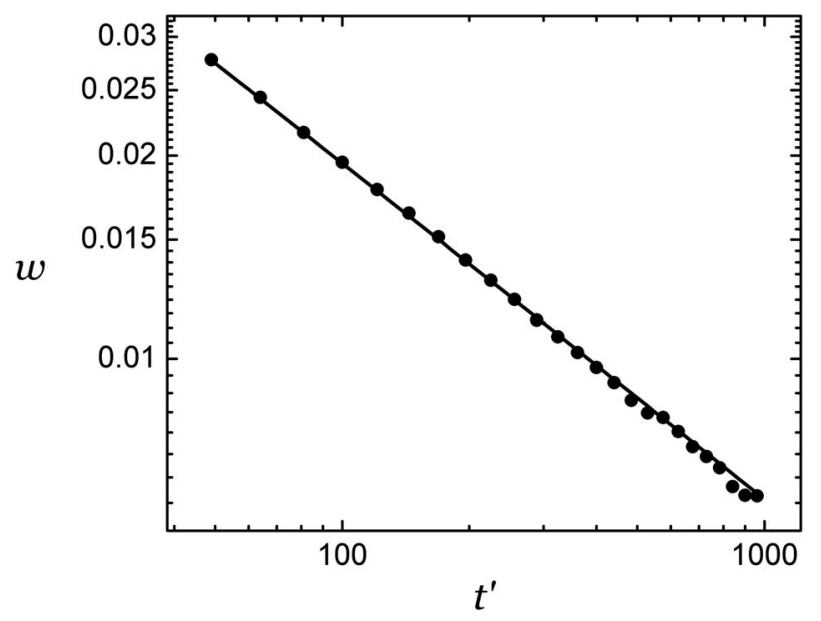

FIG. 5. Double-logarithmic plot of the half width or standard deviation $w$ of the distribution $P\left(\left\langle v^{\prime 2}\right\rangle\right)$ (see Fig. 4) as a function of $t^{\prime}$. The straight line corresponds to $w=k t^{\prime-1 / 2}$, where $k$ is a constant.

allowed and the equilibrium statistics is determined by the Boltzmann weight $e^{-\mathcal{H} / k_{B} T}$, where $\mathcal{H}$ is the bending energy (7). The bending energy is clearly minimized if the polymer configuration is a straight line parallel to the symmetry axis of the channel. In the limit $D \ll P \ll L$ the free energy of confinement and the extension again are given by Eqs. (1) and (2), as shown below, but with different amplitudes $A_{\mathcal{S}}$ and $\alpha_{\mathcal{S}}$, which we determine.

In the regime $D \ll P \ll L$, typical configurations of a semiflexible polymer correspond to single-valued functions $\vec{r}(t)$ $=\hat{r}(t) R$, where $\vec{r}$ specifies the transverse displacement of the polymer from the symmetry axis or $t$ axis of the channel. Here $(r, \theta, t)$ are cylindrical coordinates and $\hat{r}, \hat{\theta}, \hat{t}$ are the corresponding unit vectors. Keeping in mind that $d \hat{r} / d \theta=\hat{\theta}$, $d \hat{\theta} / d \theta=-\hat{r}$, and $|R d \theta / d t| \ll 1$, one finds that the bending energy (7) takes the form

$$
\mathcal{H}=\frac{\kappa}{2} \int_{0}^{L} d t\left[\left(R \frac{d^{2} \theta}{d t^{2}}\right)^{2}+\frac{1}{R^{2}}\left(R \frac{d \theta}{d t}\right)^{4}\right] .
$$

As discussed below Eq. (11), the polymer configuration $\vec{r}(t)$ may be interpreted as the position of a randomly accelerated particle in the $(x, y)$ plane, plotted as a function of time. A polymer confined to the surface of a cylinder corresponds to a particle moving on a circle of radius $R$. The first and second terms in the integrand in Eq. (32) are the squares of the tangential and centripetal accelerations, respectively.

Equation (32) and the definitions $x=R \theta=\frac{1}{2} D \theta$ and $v$ $=\frac{1}{2} D d \theta / d t$ lead to the partition function

$$
\begin{aligned}
& Z\left(x-x_{0}, v, v_{0}, t\right) \\
& =\int D x \exp \left\{-\frac{P}{2} \int_{0}^{t} d t\left[\left(\frac{d^{2} x}{d t^{2}}\right)^{2}+\frac{4}{D^{2}}\left(\frac{d x}{d t}\right)^{4}\right]\right\}
\end{aligned}
$$

and the Fokker-Plack equation

$$
\left(\frac{\partial}{\partial t}+v \frac{\partial}{\partial x}+\frac{2 P}{D^{2}} v^{4}-\frac{1}{2 P} \frac{\partial^{2}}{\partial v^{2}}\right) Z\left(x-x_{0}, v, v_{0}, t\right)=0 .
$$

In contrast to the case of a polymer in a channel, the variable $x$ in Eq. (34) is not restricted to a finite interval, but varies from $-\infty$ to $\infty$. Instead of the hard wall boundary condition, the solution to Eq. (34) satisfies $\lim _{x \rightarrow \pm \infty} Z(x$ $\left.-x_{0}, v, v_{0}, t\right)=0$. These simplifying features enable us to calculate $A_{\mathcal{S}}$ and $\alpha_{\mathcal{S}}$ with an analytic approach, without recourse to a simulation.

Disregarding the position of the polymer end point, we integrate Eq. (34) over $x$ from $-\infty$ to $\infty$ and impose the boundary condition mentioned in the preceding paragraph. This yields the Schrödinger equation

$$
\left(\frac{\partial}{\partial t}+\frac{2 P}{D^{2}} v^{4}-\frac{1}{2 P} \frac{\partial^{2}}{\partial v^{2}}\right) Z\left(v, v_{0}, t\right)=0 .
$$

Equation (35) also follows directly from the path integral $Z\left(v, v_{0}, t\right)=\int D v \exp \left\{-\frac{1}{2} P \int_{0}^{t} d t\left[(d v / d t)^{2}+\left(4 / D^{2}\right) v^{4}\right]\right\}$, which has the same Boltzmann factor as in Eq. (33), but expressed in terms of $v$ rather than $x$.

Beginning with Eq. (35) and following the steps that led from Eq. (11) to Eqs. (18) and (21), we obtain

$$
A_{\mathcal{S}}=2^{-1 / 3} E_{0}^{\mathcal{S}}\left(\frac{1}{2}, 1\right), \quad \alpha_{\mathcal{S}}=2^{-5 / 3}\left\langle v^{\prime 2}\right\rangle_{1 / 2,1}^{\mathcal{S}} .
$$

Here $E_{0}^{\mathcal{S}}\left(\frac{1}{2}, 1\right)$ is the ground-state energy of the Schrödinger equation with a quartic potential

$$
\left(-E^{\mathcal{S}}+v^{\prime 4}-\frac{\partial^{2}}{\partial v^{\prime 2}}\right) \psi\left(v^{\prime}\right)=0,
$$

written in terms of the dimensionless variables introduced below Eq. (17), $\psi_{0}\left(v^{\prime}\right)=\psi_{0}\left(-v^{\prime}\right)$ is the wave function of the ground state, and $\left\langle v^{\prime 2}\right\rangle_{1 / 2,1}^{\mathcal{S}}$ is the quantum-mechanical expectation value of $v^{\prime 2}$ in the ground state.

The ground-state energy of Eq. (37), determined numerically by Bender et al. [22] and Voros [23], is given by $E_{0}^{\mathcal{S}}\left(\frac{1}{2}, 1\right)=1.06036209$. Solving the Schrödinger equation (37) numerically for this energy using Mathematica, we obtain $\left\langle v^{\prime 2}\right\rangle_{1 / 2,1}^{\mathcal{S}}=0.362023$. Substitution of these values in Eq. (36) yields the amplitudes

$$
A_{\mathcal{S}}=0.84161, \quad \alpha_{\mathcal{S}}=0.11403 .
$$

\section{CONCLUDING REMARKS}

Using a PERM-inspired simulation algorithm, we have determined the amplitudes $A_{\square}, A_{\circ}, \alpha_{\square}$, and $\alpha_{\circ}$ for a semiflexible polymer in a channel with an estimated error of less than a tenth of a percent. We hope the results will be useful in analyzing experiments. For a polymer confined to the surface of a cylinder, we have calculated the corresponding amplitudes $A_{\mathcal{S}}$ and $\alpha_{\mathcal{S}}$ exactly to five significant figures with an analytical approach. These latter results may be used as a benchmark for testing simulation algorithms.

A common approximation in studies of semiflexible polymers in channels is to replace the hard wall interaction by an 
effective parabolic potential $[21,24]$. In concluding, we use this approximation to relate the free energy and extension amplitudes $A$ and $\alpha$ and compare the relations with our simulation results.

The partition function of a polymer which is tightly confined along the $t$ axis by a parabolic potential energy per unit length $u=\frac{1}{2} k_{B} T b \vec{r}^{2}$ is given by the path integral

$$
Z\left(\vec{r}, \vec{v} ; \vec{r}_{0}, \vec{v}_{0} ; t\right)=\int D^{2} r \exp \left\{-\frac{1}{2} \int_{0}^{t} d t\left[P\left(\frac{d^{2} \vec{r}}{d t^{2}}\right)^{2}+b \vec{r}^{2}\right]\right\} \text {. }
$$

It can be calculated exactly $[21,24]$ and yields

$$
\begin{aligned}
f(b, P) & =k_{B} T\left(\frac{4 b}{P}\right)^{1 / 4}, \\
\left\langle\vec{v}^{2}\right\rangle & =\left(4 b P^{3}\right)^{-1 / 4},
\end{aligned}
$$

where $f=-k_{B} T \lim _{t \rightarrow \infty} t^{-1} \ln Z$ is the free energy per unit length and $\left\langle\vec{v}^{2}\right\rangle$ is evaluated in the same limit $t \rightarrow \infty$.

For a parabolic potential the confinement free energy per unit length is $\Delta f=f(b, P)-f(0, P)=f(b, P)$. To obtain a prediction for $\alpha_{\circ}$, we choose $b$ so that this $\Delta f$ exactly reproduces expression (1) for the free energy of confinement in a channel with a circular cross section, evaluate $\left\langle\vec{v}^{2}\right\rangle$ in Eq. (41) for this $b$, and then substitute the result into Eq. (19). This and a similar calculation for a channel with a square cross section yield

$$
\alpha_{\square} A_{\square}=\frac{1}{8}, \quad \alpha_{\circ} A_{\circ}=\frac{1}{2} .
$$

Ubbink and Odijk [25] argue that the parabolic confining potential is an artifice and that the confinement free energy (1) of the polymer in a channel should not be identified with the full free energy $f$ in Eq. (40) but with the configurational part $f_{\text {config }}=f-\langle u\rangle=f-b \partial f / \partial b=\frac{3}{4} f$. This leads to [26]

$$
\alpha_{\square} A_{\square}=\frac{3}{32}, \quad \alpha_{\circ} A_{\circ}=\frac{3}{8},
$$

which differs from Eq. (42) by an extra factor of $\frac{3}{4}$ on the right-hand side. Our simulation results in Eqs. (30), (31), and (38) yield the products

$$
\begin{gathered}
\alpha_{\square} A_{\square}=0.1009 \pm 0.0002, \quad \alpha_{\circ} A_{\circ}=0.4008 \pm 0.0003, \\
\alpha_{\mathcal{S}} A_{\mathcal{S}}=0.095969,
\end{gathered}
$$

which lie in between the predictions (42) and (43) but in every case closer to Eqs. (43). Since the surface of a cylinder is two dimensional, the product $\alpha_{\mathcal{S}} A_{\mathcal{S}}$ should be compared with the predictions for $\alpha_{\square} A_{\square}$ in Eqs. (42) and (43), which apply in both two and three dimensions.

\section{ACKNOWLEDGMENTS}

We thank Hsiao-Ping Hsu, Walter Nadler, and Roland Winkler for helpful discussions. Hsiao-Ping Hsu showed us how to set up a PERM program for the polymer on the cylinder and has confirmed some of our results. T.W.B. greatly appreciates the hospitality of the theory group for Soft Matter and Biophysics at the Forschungszentrum Jülich. Y.Y. acknowledges financial support from the International Helmholtz Research School "BioSoft."
[1] T. Odijk, Macromolecules 16, 1340 (1983); 19, 2313 (1986).

[2] W. Reisner, K. J. Morton, R. Riehn, Y. M. Wang, Z. Yu, M. Rosen, J. C. Sturm, S. Y. Chou, E. Frey, and R. H. Austin, Phys. Rev. Lett. 94, 196101 (2005).

[3] S. Köster, D. Steinhauser, and T. Pfohl, J. Phys.: Condens. Matter 17, S4091 (2005).

[4] M. B. Hochrein, J. A. Leierseder, L. Golubovic, and J. O. Rädler, Phys. Rev. Lett. 96, 038103 (2006).

[5] T. W. Burkhardt, J. Phys. A 30, L167 (1997).

[6] D. J. Bicout and T. W. Burkhardt, J. Phys. A 34, 5745 (2001).

[7] M. Dijkstra, D. Frenkel, and H. N. W. Lekkerkerker, Physica A 193, 374 (1993).

[8] J. Wang and H. Gao, J. Chem. Phys. 123, 084906 (2005).

[9] J. Z. Y. Chen and D. E. Sullivan, Macromolecules 39, 7769 (2006).

[10] A. Lamura, T. W. Burkhardt, and G. Gompper, Phys. Rev. E 70, 051804 (2004).

[11] D. J. Bicout and T. W. Burkhardt, J. Phys. A 33, 6835 (2000).

[12] S. N. Kotsev and T. W. Burkhardt, Phys. Rev. E 71, 046115 (2005).

[13] B. Maier and J. O. Rädler, Phys. Rev. Lett. 82, 1911 (1999).

[14] B. Maier and J. O. Rädler, Macromolecules 33, 7185 (2000).
[15] P. Grassberger, Phys. Rev. E 56, 3682 (1997).

[16] P. Grassberger, H. Frauenkron, and W. Nadler, in Monte Carlo Approach to Biopolymers and Protein Folding, edited by P. Grassberger, G. Barkema, and W. Nadler (World Scientific, Singapore, 1998), p. 301.

[17] P. Grassberger and W. Nadler, in Computational Statistical Physics-From Billiards to Monte Carlo, edited by K. H. Hoffmann and M. Schreiber (Springer, Berlin, 2002), p. 169.

[18] T. Odijk, J. Chem. Phys. 125, 204904 (2006).

[19] H. Frauenkron, M. S. Causo, and P. Grassberger, Phys. Rev. E 59, R16 (1999).

[20] H.-P. Hsu and P. Grassberger, Eur. Phys. J. B 36, 209 (2003).

[21] P. Levi and K. Mecke, Europhys. Lett. 78, 38001 (2007).

[22] C. M. Bender, K. Olaussen, and P. S. Wang, Phys. Rev. D 16, 1740 (1977).

[23] A. Voros, J. Phys. A 27, 4653 (1994).

[24] T. W. Burkhardt, J. Phys. A 28, L629 (1995).

[25] J. Ubbink and T. Odijk, Biophys. J. 76, 2502 (1999).

[26] K. Jo, D. M. Dhingra, T. Odijk, J. J. de Pablo, M. D. Graham, R. Runnheim, D. Forrest, and D. C. Schwartz, Proc. Natl. Acad. Sci. U.S.A. 104, 2673 (2007). 